\title{
Ehrhart polynomials of polytopes and spectrum at infinity of Laurent polynomials
}

\author{
Antoine Douai \\ Université Côte d'Azur, CNRS, LJAD, FRANCE \\ Email address: antoine.douai@univ-cotedazur.fr
}

December 12, 2018

\begin{abstract}
Gathering different results from singularity theory, geometry and combinatorics, we show that the spectrum at infinity of a tame Laurent polynomial counts lattice points in polytopes and we deduce an effective algorithm in order to compute the Ehrhart polynomial of a simplex containing the origin as an interior point.
\end{abstract}

\section{Introduction}

The spectrum at infinity of a tame Laurent polynomial $f$, defined by C. Sabbah in [13], is a sequence $\left(\beta_{1}, \cdots, \beta_{\mu}\right)$ of rational numbers which is related to various concepts in singularity theory (monodromy, Hodge and Kashiwara-Malgrange filtrations, Brieskorn lattices...) and is in general difficult to handle. However, if $f$ is convenient and nondegenerate with respect to its Newton polytope in the sense of Kouchnirenko [10] (this is generically the case), this spectrum has a very concrete description: the sum $\sum_{i=1}^{\mu} z^{\beta_{i}}$ is equal to the Hilbert-Poincare series of the Jacobian ring of $f$ graded by the Newton filtration, see [7]. It follows from [10] that $\sum_{i=1}^{\mu} z^{\beta_{i}}=(1-z)^{n} \sum_{v \in N} z^{\nu(v)}$ where $\nu$ is the Newton filtration of the Newton polytope $P$ of $f$ and $\mu$ is the normalized volume of $P$, see [5], 6]. Because the right hand side depends only on $P$, we will also call it the Newton

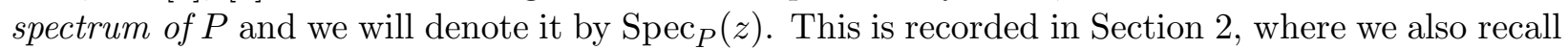
the basic definitions.

Once we have this description, it follows from the work of A. Stapledon [14 that the spectrum at infinity of a convenient and nondegenerate Laurent polynomial (equivalently, the Newton spectrum of its Newton polytope) counts "weighted" lattice points in its Newton polytope $P$ and its integer dilates, more precisely that it is a weighted $\delta$-vector as defined in loc. cit. In particular, according to an observation made in [14], the $\delta$-vector $\delta_{P}(z)=\delta_{0}+\delta_{1} z+\cdots+\delta_{n} z^{n}$ of a lattice polytope $P$ in $\mathbb{R}^{n}$ containing the origin as an interior point, hence its Ehrhart polynomial, can be obtained effortlessly from its Newton spectrum $\sum_{i=1}^{\mu} z^{\beta_{i}}$ : the coefficient $\delta_{i}$ is equal to the number of $\beta_{k}$ 's such that $\left.\left.\beta_{k} \in\right] i-1, i\right]$. See Section 4.1, where we also recall the definitions of $\delta$-vectors and Ehrhart polynomials. It should be emphasized that the Newton spectrum of a polytope $P$ is somewhat finer than its $\delta$-vector: both coincide if and only if $P$ is reflexive, see Proposition 5.1.

This is particularly fruitful when $P$ is a reduced simplex in $\mathbb{R}^{n}$ in the sense of [4] (see Section 3 ] for the definition of a reduced simplex and its weight) because we have a very simple closed formula 
for its Newton spectrum, see Theorem 3.3. This formula only involves the weight of the simplex and is based on [8, Theorem 1] whose proof uses classical tools in singularity theory. This provides an effective algorithm in order to compute the $\delta$-vectors and the Ehrhart polynomials of reduced simplices, see Section 4.2, For instance, let us consider the simplex

$$
\Delta:=\operatorname{conv}((1,0,0,0),(0,1,0,0),(0,0,1,0),(0,0,0,1),(-1,-1,-1,-5))
$$

in $\mathbb{R}^{4}$. Using Theorem 3.3 , it is readily checked that its Newton spectrum is

$$
\operatorname{Spec}_{\Delta}(z)=1+z+z^{2}+z^{3}+z^{4}+z^{16 / 5}+z^{12 / 5}+z^{8 / 5}+z^{4 / 5}
$$

and it follows from Proposition 4.5 that $\delta_{0}=1, \delta_{1}=2, \delta_{2}=2, \delta_{3}=2, \delta_{4}=2$. Finally, its Ehrhart polynomial is

$$
L_{\Delta}(z)=\frac{1}{24}\left(9 z^{4}+10 z^{3}+75 z^{2}+50 z+24\right)
$$

See Example 4.7 .

Notice that it is also possible to get in a similar way the $\delta$-vectors of Newton polytopes of convenient and nondegenerate polynomials from their toric Newton spectrum (as defined in [ 5 , Definition 3.1]). This can be applied for instance to the Mordell-Pommersheim tetrahedron, that is the convex hull of $(0,0,0),(a, 0,0),(0, b, 0),(0,0, c))$ where $a, b$ and $c$ are positive integers, which is the Newton polytope of the Brieskorn-Pham polynomial $f\left(u_{1}, u_{2}, u_{3}\right)=u_{1}^{a}+u_{2}^{b}+u_{3}^{c}$ on $\mathbb{C}^{3}$, a very familiar object in singularity theory. See Section 4.3 .

In this context, other questions may arise: for instance it is expected that the variance of the spectrum at infinity of a Laurent polynomial in $\left(\mathbb{C}^{*}\right)^{n}$ is bounded below by $n / 12$ (this is a global variant of C. Hertling's conjecture [9], see [6] and the references therein). By Proposition 5.1, this would give informations about the distribution of the $\delta$-vector in the reflexive case. See Remark 5.3. In the opposite direction, the theory of polytopes is useful in order to get results on the singularity side: for instance, using [12] it is readily seen that the spectrum at infinity of a tame regular function is not always unimodal (this is discussed in Remark 5.2).

Acknowledgements. I thank R. Davis for pointing to me the references [3] and [12] about unimodality.

\section{The Newton spectrum of a polytope}

In this section, we first recall some basic facts about polytopes that we will use. We then define the Newton spectrum of a polytope in Section 2.2 and we give a closed formula for the Newton spectrum of simplices in Section 3 .

\subsection{Polytopes (basics)}

In this text, $N$ is the lattice $\mathbb{Z}^{n}, M$ is the dual lattice $\langle$,$\rangle is the canonical pairing between N_{\mathbb{R}}$ and $M_{\mathbb{R}}$. A lattice polytope is the convex hull of a finite set of $N$. If $P \subset N_{\mathbb{R}}$ is a $n$-dimensional lattice polytope containing the origin as an interior point there exists, for each facet $F$ of $P, u_{F} \in M_{\mathbb{Q}}$ such that

$$
P \subset\left\{n \in N_{\mathbb{R}},\left\langle u_{F}, n\right\rangle \leq 1\right\} \text { and } F=P \cap\left\{x \in N_{\mathbb{R}},\left\langle u_{F}, x\right\rangle=1\right\} .
$$


This provides the hyperplane presentation

$$
P=\cap_{F}\left\{x \in N_{\mathbb{R}},\left\langle u_{F}, x\right\rangle \leq 1\right\}
$$

The set

$$
P^{\circ}:=\left\{y \in M_{\mathbb{R}},\langle y, x\rangle \leq 1 \text { for all } x \in P\right\}
$$

is the polar polytope of $P$. It happens that $P^{\circ}$ is indeed a rational polytope (the convex hull of a finite set of $N_{\mathbb{Q}}$ ) if $P$ contains the origin as an interior point: the vertices of $P^{\circ}$ are in one-to-one correspondance with the facets of $P$ by

$$
u_{F} \text { vertice of } P^{\circ} \Leftrightarrow F=\left\{x \in N_{\mathbb{R}},\left\langle u_{F}, x\right\rangle=1\right\} \text {. }
$$

A lattice polytope $P$ is reflexive if it contains the origin as an interior point and if $P^{\circ}$ is a lattice polytope.

If $P \subset N_{\mathbb{R}}$ is a full dimensional lattice polytope containing the origin as an interior point, we get a complete fan $\Sigma_{P}$ in $N_{\mathbb{R}}$ by taking the cones over the faces of $P$. We will denote by $X_{\Sigma_{P}}$ the complete toric variety associated with the fan $\Sigma_{P}$. We will always assume that the fan $\Sigma_{P}$ is simplicial. The Newton function of $P$ is the function

$$
\nu: N_{\mathbb{R}} \rightarrow \mathbb{R}
$$

which takes the value 1 at the vertices of $P$ and which is linear on each cone of the fan $\Sigma_{P}$. Alternatively, $\nu(v)=\max _{F}\left\langle u_{F}, v\right\rangle$ where the maximum is taken over the facets of $P$ and the vectors $u_{F}$ are defined as in (11). The Milnor number of $P$ is

$$
\mu_{P}:=n ! \operatorname{vol}(P)
$$

where the volume $\operatorname{vol}(P)$ is normalized such that the volume of the cube is equal to 1 . If $P=$ $\operatorname{conv}\left(b_{1}, \cdots, b_{r}\right)$, the Milnor number $\mu_{P}$ is also the global Milnor number of the Laurent polynomial $f(\underline{u})=\sum_{i=1}^{r} c_{i} \underline{u}^{b_{i}}$ for generic complex coefficients $c_{i}$, that is

$$
\mu_{P}=\operatorname{dim}_{\mathbb{C}} \frac{\mathbb{C}\left[u_{1}, u_{1}^{-1}, \cdots, u_{n}, u_{n}^{-1}\right]}{\left(u_{1} \frac{\partial f}{\partial u_{1}}, \cdots, u_{n} \frac{\partial f}{\partial u_{n}}\right)}
$$

where $\left(\mathbb{C}^{*}\right)^{n}$ is equipped with the coordinates $\underline{u}=\left(u_{1}, \cdots, u_{n}\right), \underline{u}^{m}:=u_{1}^{m_{1}} \cdots u_{n}^{m_{n}}$ if $m=$ $\left(m_{1}, \cdots, m_{n}\right) \in \mathbb{Z}^{n}$ and $\left(u_{1} \frac{\partial f}{\partial u_{1}}, \cdots, u_{n} \frac{\partial f}{\partial u_{n}}\right)$ denotes the ideal generated by the partial derivative $u_{1} \frac{\partial f}{\partial u_{1}}, \cdots, u_{n} \frac{\partial f}{\partial u_{n}}$. See [10] for details.

\subsection{The Newton spectrum of a polytope}

The main object of this paper is given by Definition 2.3 which is motivated by the description of the spectrum at infinity of a Laurent polynomial in Kouchnirenko's framework, see [5], 6], [7], 13]. We briefly recall the construction and we gather the results that we will use.

Let $f(\underline{u})=\sum_{m \in \mathbb{Z}^{n}} a_{m} \underline{u}^{m}$ be a Laurent polynomial defined on $\left(\mathbb{C}^{*}\right)^{n}$. The Newton polytope $P$ of $f$ is the convex hull of $\operatorname{supp} f:=\left\{m \in \mathbb{Z}^{n}, a_{m} \neq 0\right\}$ in $\mathbb{R}^{n}$. We assume that $P$ contains the origin as an interior point, so that $f$ is convenient in the sense of [10]. The Newton function of $P$ defines a filtration on $\mathcal{A}:=\mathbb{C}\left[u_{1}, u_{1}^{-1}, \cdots, u_{n}, u_{n}^{-1}\right]$ and, by projection, a filtration $\mathcal{N}_{\bullet}$ on 
$\mathcal{A} /\left(u_{1} \frac{\partial f}{\partial u_{1}}, \cdots, u_{n} \frac{\partial f}{\partial u_{n}}\right)$, see [5, Section2], [7, Section 4]. The spectrum at infinity of $f$ is a priori a sequence $\left(\beta_{1}, \cdots, \beta_{\mu_{P}}\right)$ of nonnegative rational numbers (there may be repeated items), see [13], [7, Section 2.e]. We will denote $\operatorname{Spec}_{f}(z):=\sum_{i=1}^{\mu_{P}} z^{\beta_{i}}$. We first quote the following result (for our purpose, one can take equality (4) as the definition of the spectrum at infinity of the Laurent polynomial $f$ ) which holds if $f$ is moreover nondegenerate with respect to $P$ in the sense of [10, Définition 1.19]:

Theorem 2.1 [7] Let $f$ be a convenient and nondegenerate Laurent polynomial defined on $\left(\mathbb{C}^{*}\right)^{n}$. Then

$$
\operatorname{Spec}_{f}(z)=\sum_{\alpha \in \mathbb{Q}} \operatorname{dim}_{\mathbb{C}} \operatorname{gr}_{\alpha}^{\mathcal{N}} \frac{\mathcal{A}}{\left(u_{1} \frac{\partial f}{\partial u_{1}}, \cdots, u_{n} \frac{\partial f}{\partial u_{n}}\right)} z^{\alpha} .
$$

Proof. By [7, Theorem 4.5], the Newton filtration and the Kashiwara-Malgrange filtration on the Brieskorn lattice of $f$ coincide if $f$ is convenient and nondegenerate. The result then follows from the description of the spectrum given in [7, Section 2.e].

Corollary 2.2 [5], [6] Let $f$ be a convenient and nondegenerate Laurent polynomial defined on $\left(\mathbb{C}^{*}\right)^{n}$. Then

$$
\operatorname{Spec}_{f}(z)=(1-z)^{n} \sum_{v \in N} z^{\nu(v)}
$$

where $\nu$ is the Newton function of the Newton polytope $P$ of $f$.

Proof. Follows from Theorem 2.1 and [10, Théorème 2.8], as in [5, Theorem 3.2],

The right hand side of (5) depends only on $P$. This justifies the following definition:

Definition 2.3 Let $P$ be a full dimensional lattice polytope in $N_{\mathbb{R}}$, containing the origin as an interior point, and let $\nu$ be its Newton function. The Newton spectrum of $P$ is

$$
\operatorname{Spec}_{P}(z):=(1-z)^{n} \sum_{v \in N} z^{\nu(v)}
$$

Notice that various versions of the right hand of (6) appear in many places, see for instance [1, Theorem 4.3] for a relation with stringy $E$-functions.

We have the following geometric description of the Newton spectrum of a polytope $P$, which allows to identify the spectrum at infinity of a convenient and nondegenerate polynomial whose Newton polytope is $P$. Define, for $\sigma$ a cone in the fan $\Sigma_{P}$ generated by the vertices $b_{1}, \cdots, b_{r}$ of $P$,

$$
\square(\sigma):=\left\{\sum_{i=1}^{r} q_{i} b_{i}, q_{i} \in[0,1[, i=1, \cdots, r\}\right.
$$

and $\square\left(\Sigma_{P}\right):=\cup_{\sigma \in \Sigma_{P}} \square(\sigma)$. 
Proposition 2.4 [5], [6], [14] Let $P$ be a full dimensional lattice polytope in $N_{\mathbb{R}}$, containing the origin as an interior point. Then

$$
\operatorname{Spec}_{P}(z)=\sum_{v \in \square\left(\Sigma_{P}\right) \cap N}\left[\sum_{i=0}^{n-\operatorname{dim} \sigma(v)} \operatorname{dim} H^{2 i}\left(X\left(\Sigma_{P} / \sigma(v)\right), \mathbb{Q}\right) z^{i}\right] z^{\nu(v)}
$$

where $X\left(\Sigma_{P} / \sigma(v)\right)$ is the toric variety associated with the quotient fan $\Sigma_{P} / \sigma(v)$ and $\sigma(v)$ is the smallest cone containing $v$.

Proof. Follows from [10, Proposition 2.6], as in [5, Proposition 3.3]. See also [14, Theorem 4.3] for another proof in a slightly different context.

Remark 2.5 We will write $\operatorname{Spec}_{P}(z):=\sum_{i=1}^{\mu_{P}} z^{\beta_{i}}$ where $\left(\beta_{1}, \cdots \beta_{\mu_{P}}\right)$ is a sequence of nonnegative rational numbers (counted with multiplicities) and we will freely identify the Newton spectrum of $P$ with the sequence $\left(\beta_{1}, \cdots \beta_{\mu_{P}}\right)$.

We will make a repeated use of the following properties. In what follows, Int $P$ denotes the interior of $P, \partial P$ denotes its boundary and, for $I \subset \mathbb{R}, \operatorname{Spec}_{P}^{I}(z):=\sum_{\beta_{i} \in I} z^{\beta_{i}}$.

Proposition 2.6 [5], [6], [7] Let $P$ be a full dimensional polytope in $N_{\mathbb{R}}$ containing the origin in its interior and let $\nu$ be its Newton function. Then,

1. $\lim _{z \rightarrow 1} \operatorname{Spec}_{P}(z)=\mu_{P}$,

2. $\operatorname{Spec}_{P}^{[0,1[}(z)=\sum_{v \in \operatorname{Int} P \cap N} z^{\nu(v)}$,

3. the multiplicity of 1 in $\operatorname{Spec}_{P}(z)$ is $\operatorname{Card}(\partial P \cap N)-n$,

4. $z^{n} \operatorname{Spec}_{P}\left(z^{-1}\right)=\operatorname{Spec}_{P}(z)$.

See [5, Corollary 3.5]. Item 1, 2 and 4 are already contained in [7] and are based on Kouchnirenko's results [10]. The last property means that $\operatorname{Spec}_{P}$ is symmetric about $\frac{n}{2}$ and is a classical specification of a singularity spectrum. In a different setting, these statements can also be found in [14].

\section{The Newton spectrum of a reduced simplex}

We keep the terminology of [4] and we will rather denote a simplex by $\Delta$ instead of $P$. We will say that the polytope $\Delta:=\operatorname{conv}\left(v_{0}, \cdots, v_{n}\right)$ is an integral $\mathbb{Z}^{n}$-simplex if its vertices $v_{i}$ belong to the lattice $\mathbb{Z}^{n}$ and if it contains the origin as an interior point.

Definition 3.1 Let $\Delta:=\operatorname{conv}\left(v_{0}, \cdots, v_{n}\right)$ be an integral $\mathbb{Z}^{n}$-simplex. The weight of $\Delta$ is the tuple $Q(\Delta)=\left(q_{0}, \cdots, q_{n}\right)$ where

$$
q_{i}:=\left|\operatorname{det}\left(v_{0}, \cdots, \widehat{v}_{i}, \cdots, v_{n}\right)\right|
$$

for $i=0, \cdots, n$. The simplex $\Delta$ is said to be reduced if $\operatorname{gcd}\left(q_{0}, \cdots, q_{n}\right)=1$. 
Proposition 3.2 Let $\Delta:=\operatorname{conv}\left(v_{0}, \cdots, v_{n}\right)$ be an integral $\mathbb{Z}^{n}$-simplex and let $Q(\Delta)=\left(q_{0}, \cdots, q_{n}\right)$ be its weight. Then,

1. $\mu_{\Delta}=\sum_{i=0}^{n} q_{i}$ where $\mu_{\Delta}$ is the Milnor number of $\Delta$,

2. $\sum_{i=0}^{n} q_{i} v_{i}=0$,

3. $\left(v_{0}, \cdots, v_{n}\right)$ generate $N$ if and only if $\Delta$ is reduced.

Proof. The first point follows from the definition of the $q_{i}$ 's and the second one follows from Cramer's rule. For the third one notice first that, because $q_{i}>0$, the submodule generated by $\left(v_{0}, \cdots, \widehat{v}_{i}, \cdots, v_{n}\right)$ is free of rank $n$, hence the module $N_{\Delta}$ generated by $\left(v_{0}, \cdots, v_{n}\right)$ is free of rank $n$. By [4, Lemma 2.4] we have $\operatorname{det} N_{\Delta}=\operatorname{gcd}\left(q_{0}, \cdots, q_{n}\right)$ and this is the index of $N_{\Delta}$ in $N$ so that $N_{\Delta}=N$ if and only if $\operatorname{det} N_{\Delta}=1$.

Let $\Delta$ be a $\mathbb{Z}^{n}$-integral simplex and let $Q(\Delta)=\left(q_{0}, \cdots, q_{n}\right)$ be its weight. Let

$$
F:=\left\{\frac{\ell}{q_{i}} \mid 0 \leq \ell \leq q_{i}-1,0 \leq i \leq n\right\}
$$

and let $f_{1}, \cdots, f_{k}$ be the elements of $F$ arranged by increasing order. Define

$$
S_{f_{i}}:=\left\{j \mid q_{j} f_{i} \in \mathbb{Z}\right\} \subset\{0, \cdots, n\} \text { and } d_{i}:=\operatorname{Card} S_{f_{i}}
$$

and let $c_{0}, c_{1}, \cdots, c_{\mu_{\Delta}-1}$ be the sequence

$$
\underbrace{f_{1}, \cdots, f_{1}}_{d_{1}}, \underbrace{f_{2}, \cdots, f_{2}}_{d_{2}}, \cdots, \underbrace{f_{k}, \cdots, f_{k}}_{d_{k}} .
$$

Theorem 3.3 Let $\Delta$ be a $\mathbb{Z}^{n}$-integral simplex and let $Q(\Delta)=\left(q_{0}, \cdots, q_{n}\right)$ be its weight. Assume that $\Delta$ is reduced. Then the Newton spectrum of $\Delta$ is

$$
\operatorname{Spec}_{\Delta}(z)=z^{\alpha_{0}}+z^{\alpha_{1}}+\cdots+z^{\alpha_{\mu^{-1}}}
$$

where

$$
\alpha_{k}:=k-\mu_{\Delta} c_{k}
$$

for $k=0, \cdots, \mu_{\Delta}-1$.

Proof. The vertices $v_{0}, \cdots, v_{n}$ of $\Delta$ satisfy $\sum_{i=0}^{n} q_{i} v_{i}=0$ and they generate $\mathbb{Z}^{n}$ by Proposition 3.2 because $\operatorname{gcd}\left(q_{0}, \cdots, q_{n}\right)=1$. Thus, they define the exact sequence

$$
0 \longrightarrow \mathbb{Z} \longrightarrow \mathbb{Z}^{n+1} \longrightarrow \mathbb{Z}^{n} \longrightarrow 0
$$

where the map on the right is defined by $\psi\left(e_{i}\right)=v_{i}$ for $i=0, \cdots, n$ (we denote here by $\left(e_{0}, \cdots, e_{n}\right)$ the canonical basis of $\left.\mathbb{Z}^{n+1}\right)$ and the map on the left is defined by $\phi(1)=\left(q_{0}, \cdots, q_{n}\right)$. It follows that the simplex $\Delta$ is the Newton polytope of the convenient and nondegenerate Laurent polynomial considered in [8]. Thus, the assertion follows from the definition of the Newton spectrum of a polytope given in Section 2.2 and [8, Theorem 1]. 
Remark 3.4 The $\alpha_{k}$ 's defined in Theorem 3.3 are not necessarily distinct. For instance, let us consider $\Delta=\operatorname{conv}((1,0),(0,1),(-1,-2))$ in $\mathbb{R}^{2}$. We have $Q(\Delta)=(1,1,2), \mu_{\Delta}=4$ and the sequence $\alpha_{0}, \alpha_{1}, \alpha_{2}, \alpha_{3}$ is $0,1,2,1$.

Example 3.5 Let us consider

$$
\Delta=\operatorname{conv}((1,0,0,0),(0,1,0,0),(0,0,1,0),(0,0,0,1),(-1,-1,-1,-5))
$$

in $\mathbb{R}^{4}$. We have $Q(\Delta)=(1,1,1,1,5)$ and $\mu_{\Delta}=9$. The sequence $c_{0}, c_{1}, \cdots, c_{8}$ is

$$
0,0,0,0,0, \frac{1}{5}, \frac{2}{5}, \frac{3}{5}, \frac{4}{5}
$$

and the sequence $\alpha_{0}, \alpha_{1}, \cdots, \alpha_{8}$ is

$$
0,1,2,3,4,5-\frac{9}{5}, 6-\frac{18}{5}, 7-\frac{27}{5}, 8-\frac{36}{5} .
$$

Theorem 3.3 provides $\operatorname{Spec}_{\Delta}(z)=1+z+z^{2}+z^{3}+z^{4}+z^{16 / 5}+z^{12 / 5}+z^{8 / 5}+z^{4 / 5}$.

Remark 3.6 Theorem 3.3 is not true if we do not assume that $\Delta$ is reduced: for instance, let $\Delta=\operatorname{conv}((2,0),(0,2),(2,2))$ in $\mathbb{R}^{2}$. We have $Q(\Delta)=(4,4,4)$ and $\Delta$ is not reduced. By Proposition [2.6] we have

$$
\operatorname{Spec}_{\Delta}(z)=1+3 z^{1 / 2}+4 z+3 z^{3 / 2}+z^{2}
$$

but Theorem 3.3 would give $4+4 z+4 z^{2}$.

\section{The spectrum as a weighted $\delta$-vector and application to the computation of (weighted) Ehrhart polynomials}

In the first part of this section, we show that the Newton spectrum of a polytope counts weighted lattice points and in the second part we write down an algorithm in order to compute Ehrhart polynomials of reduced simplices.

\subsection{The Newton spectrum of a polytope as a weighted $\delta$-vector}

Once the spectrum at infinity of a Laurent polynomial is identified by the formula (5), the results of this subsection can be basically found in [14]. For the convenience of the reader we give selfcontained proofs. For the background about Ehrhart theory we refer to the book [2].

Let $P$ be a full dimensional lattice polytope in $\mathbb{R}^{n}$ and define, for a nonnegative integer $\ell$, $L_{P}(\ell):=\operatorname{Card}((\ell P) \cap M)$. Then $L_{P}$ is a polynomial in $\ell$ of degree $n$ (this is the Ehrhart polynomial) and we have

$$
\operatorname{Ehr}_{P}(z):=1+\sum_{m \geq 1} L_{P}(m) z^{m}=\frac{\delta_{0}+\delta_{1} z+\cdots+\delta_{n} z^{n}}{(1-z)^{n+1}}
$$

where the $\delta_{j}$ 's are nonnegative integers. We will call $\operatorname{Ehr}_{P}(z)$ the Ehrhart series of $P$. We will write $\delta_{P}(z):=\delta_{0}+\delta_{1} z+\cdots+\delta_{n} z^{n}$. With a slight abuse of terminology, $\delta_{P}(z)$ is called the $\delta$-vector 
of the polytope $P$. The Ehrhart polynomial of a polytope is extract from its $\delta$-vector by the means of the formula

$$
L_{P}(z)=\delta_{0}\left(\begin{array}{c}
z+n \\
n
\end{array}\right)+\delta_{1}\left(\begin{array}{c}
z+n-1 \\
n
\end{array}\right)+\cdots+\delta_{n-1}\left(\begin{array}{c}
z+1 \\
n
\end{array}\right)+\delta_{n}\left(\begin{array}{c}
z \\
n
\end{array}\right)
$$

Following [14], one defines a weighted version of the $\delta$-vectors. Let $P$ be full dimensional lattice polytope in $\mathbb{R}^{n}$, containing the origin as an interior point, and let $\nu$ be its Newton function. The weight of $v \in N$ is $\operatorname{wt}(v):=\nu(v)-\lceil\nu(v)\rceil$ where \lceil\rceil denotes the ceiling function. For $m \in \mathbb{N}$, let $L_{P}^{\alpha}(m)$ be the number of lattice points in $m P$ of weight $\alpha$ and define

$$
\delta_{P}^{\alpha}(z):=(1-z)^{n+1} \sum_{m \geq 0} L_{P}^{\alpha}(m) z^{m} .
$$

By the very definition, we have $\delta_{P}(z)=\sum_{\alpha \in]-1,0]} \delta_{P}^{\alpha}(z)$.

Definition 4.1 The weighted $\delta$-vector of the polytope $P$ is $\delta_{P}^{\mathrm{wt}}(z):=\sum_{\alpha \in]-1,0]} \delta_{P}^{\alpha}(z) z^{\alpha}$.

Theorem 4.2 Let $P$ be a full dimensional lattice polytope containing the origin as a interior point. Then,

$$
\operatorname{Spec}_{P}(z)=\delta_{P}^{\mathrm{wt}}(z)
$$

In particular, the spectrum at infinity $\operatorname{Spec}_{f}(z)$ of a convenient and nondegenerate Laurent polynomial $f$ is equal to the weighted $\delta$-vector $\delta_{P}^{\mathrm{wt}}(z)$.

Proof. Because $v \in m P \cap N$ if and only if $\nu(v) \leq m$, we get

$$
\begin{gathered}
\delta_{P}^{\mathrm{wt}}(z)=(1-z)^{n+1} \sum_{m \geq 0}\left(\sum_{v \in m P} z^{w t(v)}\right) z^{m}=(1-z)^{n+1} \sum_{m \geq 0}\left(\sum_{\nu(v) \leq m} z^{\nu(v)-\lceil v\rceil}\right) z^{m} \\
=(1-z)^{n+1} \sum_{m \geq 0}\left(\sum_{\lceil\nu(v)\rceil \leq m} z^{\nu(v)-\lceil v\rceil}\right) z^{m}=(1-z)^{n+1} \sum_{v \in N}\left(\sum_{\lceil\nu(v)\rceil \leq m} z^{m-\lceil v\rceil}\right) z^{\nu(v)} \\
=(1-z)^{n} \sum_{v \in N} z^{\nu(v)}=\operatorname{Spec}_{P}(z) .
\end{gathered}
$$

The last assertion follows from (5) and (6).

To sum up, the spectrum at infinity of a Laurent polynomial counts weighted lattice points in its Newton polytope.

Corollary 4.3 Let $\alpha \in]-1,0]$ such that $\delta_{P}^{\alpha}(z)$ is not identically equal to zero. Then, with the notations of Proposition 2.4, the rational number $\alpha$ is equal to $\nu(v)-\lceil\nu(v)\rceil$ for a suitable $v \in$ $\square\left(\Sigma_{P}\right) \cap N$ and

$$
\delta_{P}^{\alpha}(z)=c_{v}^{0} z^{\lceil\nu(v)\rceil}+\cdots+c_{v}^{n-\operatorname{dim} \sigma(v)} z^{\lceil\nu(v)\rceil+n-\operatorname{dim} \sigma(v)}
$$

where $c_{v}^{i}:=\operatorname{dim} H^{2 i}(X(\Delta / \sigma(v)), \mathbb{Q})$.

Proof. Follows from Proposition 2.4 and Theorem 4.2 . 
Corollary 4.4 Let $\alpha \in]-1,0]$. Then,

1. $\delta_{P}^{\alpha}(z)$ is a polynomial of degree at most $n$ in $z$ whose coefficients are nonnegative integers,

2. $L_{P}^{\alpha}$ is a polynomial of degree $n$ (if not identically equal to zero) and

$$
\begin{aligned}
& L_{P}^{\alpha}(z)=\delta_{0}^{\alpha}\left(\begin{array}{c}
z+n \\
n
\end{array}\right)+\delta_{1}^{\alpha}\left(\begin{array}{c}
z+n-1 \\
n
\end{array}\right)+\cdots+\delta_{n-1}^{\alpha}\left(\begin{array}{c}
z+1 \\
n
\end{array}\right)+\delta_{n}^{\alpha}\left(\begin{array}{c}
z \\
n
\end{array}\right) \\
& \text { if } \delta_{P}^{\alpha}(z)=\delta_{0}^{\alpha}+\delta_{1}^{\alpha} z+\cdots+\delta_{n}^{\alpha} z^{n} .
\end{aligned}
$$

Proof. The first assertion follows from Corollary 4.3 because where $c_{v}^{i}:=\operatorname{dim} H^{2 i}(X(\Delta / \sigma(v)), \mathbb{Q})$ is a nonnegative integer and because $\lceil\nu(v)\rceil \leq \operatorname{dim} \sigma(v)$ (recall that the Newton spectrum is contained in $[0, n]$, see Proposition 2.6). The second one is straightforward.

The next observation is borrowed from [14]:

Proposition 4.5 Let $P$ be a full dimensional lattice polytope containing the origin as a interior point. Let $\delta_{P}(z):=\sum_{k=0}^{n} \delta_{k} z^{k}$ be the $\delta$-vector of the $P$ and let $\operatorname{Spec}_{P}(z):=\sum_{i=1}^{\mu_{P}} z^{\beta_{i}}$ be its Newton spectrum. Then, for $k=0, \cdots, n$, the coefficient $\delta_{k}$ is equal to the number of $\beta_{i}$ 's, $1 \leq i \leq \mu_{P}$, such that $\left.\left.\beta_{i} \in\right] k-1, k\right]$.

Proof. The result follows from Corollary 4.3 because $\delta_{P}(z)=\sum_{\alpha \in]-1,0]} \delta_{P}^{\alpha}(z)$.

Using Proposition 2.6. we check that $\delta_{0}=1, \delta_{1}=\operatorname{Card}(P \cap N)-(n+1)$ and $\delta_{0}+\cdots+\delta_{n}=\mu_{P}$ where $\mu_{P}$ is the Milnor number of $P$.

\subsection{An algorithm to compute (weighted) Ehrhart polynomials and $\delta$-vectors of reduced simplices}

Let $P$ be a full dimensional lattice polytope in $\mathbb{R}^{n}$ containing the origin in its interior. A general recipe in order to compute the $\delta$-vector of $P$ is now clear: compute the Newton spectrum of $P$ and use Proposition 4.5.

This provides an effective algorithm in order to compute the (weighted) $\delta$-vectors and the (weighted) Ehrhart polynomials of a reduced simplex $\Delta$ containing the origin as an interior point because the Newton spectrum of $\Delta$ is known:

- compute the weight of $\Delta$ (use equation (8) ) and check that $\Delta$ is reduced,

- compute the Newton spectrum of $\Delta$ using Theorem 3.3 ,

- use Theorem 4.2, Corollary 4.4 and equation (11) in order to compute the weighted $\delta$-vectors $\delta_{\Delta}^{\alpha}(z)$ and weighted Ehrhart polynomials $L_{\Delta}^{\alpha}(z)$,

- use Proposition 4.5 in order to get the $\delta$-vector $\delta_{\Delta}(z):=\delta_{0}+\delta_{1} z+\cdots+\delta_{n} z^{n}$ of $\Delta$ and formula (10) in order to get the Ehrhart polynomial $L_{\Delta}(z)$ of $\Delta$. 
Example 4.6 Let

$$
\Delta:=\operatorname{conv}((1,0,0),(0,2,0),(1,1,1),(-3,-5,-2))
$$

in $\mathbb{R}^{3}$. Its weight is $Q(\Delta)=(2,2,3,4)$ and $\Delta$ is reduced. We have $\mu_{\Delta}=11$ and

$$
\operatorname{Spec}_{\Delta}(z)=1+z+z^{2}+z^{3}+z^{5 / 4}+z^{4 / 3}+z^{1 / 2}+z^{3 / 2}+z^{5 / 2}+z^{5 / 3}+z^{7 / 4}
$$

by Theorem 3.3. Proposition 4.5 provides $\delta_{0}=1, \delta_{1}=2, \delta_{2}=6, \delta_{3}=2$ and we get

$$
L_{\Delta}(z)=\frac{1}{6}\left(11 z^{3}+6 z^{2}+13 z+6\right) .
$$

Example 4.7 (Example 3.5 continued) Let us consider the simplex

$$
\Delta:=\operatorname{conv}((1,0,0,0),(0,1,0,0),(0,0,1,0),(0,0,0,1),(-1,-1,-1,-5))
$$

in $\mathbb{R}^{4}$. Its weight is $Q(\Delta)=(1,1,1,1,5)$ and $\Delta$ is reduced. We have $\mu_{\Delta}=9$ and

$$
\operatorname{Spec}_{\Delta}(z)=1+z+z^{2}+z^{3}+z^{4}+z^{16 / 5}+z^{12 / 5}+z^{8 / 5}+z^{4 / 5}
$$

by Example 3.5. Proposition 4.5 provides $\delta_{0}=1, \delta_{1}=2, \delta_{2}=2, \delta_{3}=2, \delta_{4}=2$ and we get

$$
L_{\Delta}(z)=\frac{1}{24}\left(9 z^{4}+10 z^{3}+75 z^{2}+50 z+24\right) \text {. }
$$

We have also $\delta_{\Delta}^{0}(z)=1+z+z^{2}+z^{3}+z^{4}, \delta_{\Delta}^{-1 / 5}(z)=z, \delta_{\Delta}^{-2 / 5}(z)=z^{2}, \delta_{\Delta}^{-3 / 5}(z)=z^{3}, \delta_{\Delta}^{-4 / 5}(z)=z^{4}$ and

$$
\begin{gathered}
L_{\Delta}^{-1 / 5}(z)=\frac{1}{24}\left(z^{4}+6 z^{3}+11 z^{2}+6 z\right), L_{\Delta}^{-2 / 5}(z)=\frac{1}{24}\left(z^{4}+2 z^{3}-z^{2}-2 z\right), \\
L_{\Delta}^{-3 / 5}(z)=\frac{1}{24}\left(z^{4}-2 z^{3}-z^{2}+2 z\right), L_{\Delta}^{-4 / 5}(z)=\frac{1}{24}\left(z^{4}-6 z^{3} 11 z^{2}-6 z\right), \\
L_{\Delta}^{0}(z)=\frac{1}{24}\left(5 z^{4}+10 z^{3}+55 z^{2}+50 z+24\right) .
\end{gathered}
$$

In particular, weighted Ehrhart polynomials may have negative coefficients.

If $q_{0}=1$ and if $\Delta$ is reflexive, another formula (with a different proof) for the $\delta$-vector of $\Delta$ in terms of the entries of the vector $Q(\Delta)$ is given in [3, Theorem 2.2].

\subsection{Ehrhart polynomials of Newton polytopes of polynomials}

We give a few words about Newton polytopes of polynomials (and not Laurent polynomials). We briefly recall the framework. The support of a polynomial $g=\sum_{m \in \mathbb{N}^{n}} a_{m} u^{m} \in \mathbb{C}\left[u_{1}, \cdots, u_{n}\right]$, where we write $u^{m}:=u_{1}^{m_{1}} \cdots u_{n}^{m_{n}}$ if $m=\left(m_{1}, \cdots, m_{n}\right) \in \mathbb{N}^{n}$, is $\operatorname{supp}(g)=\left\{m \in \mathbb{N}^{n}, a_{m} \neq 0\right\}$ and the Newton polytope of the polynomial $g$ is the convex hull of $\{0\} \cup \operatorname{supp}(g)$ in $\mathbb{R}_{+}^{n}$. The toric Newton spectrum of $g$ (or the toric Newton spectrum of its Newton polytope) is

$$
\sum_{\alpha \in \mathbb{Q}} \operatorname{dim}_{\mathbb{C}} \operatorname{gr}_{\alpha}^{\mathcal{N}} \frac{\mathbb{C}\left[u_{1}, \cdots, u_{n}\right]}{\left(u_{1} \frac{\partial g}{\partial u_{1}}, \cdots, u_{n} \frac{\partial g}{\partial u_{n}}\right)} z^{\alpha},
$$

see [5, Definition 3.1]. Thanks to [5, Theorem 3.2], Theorem 4.2 and its corollaries (in particular Proposition 4.5) still hold, with the same proofs, for the toric Newton spectrum of the Newton polytope of a convenient and nondegenerate polynomials. This gives a recipe in order to calculate Ehrahrt polynomials of Newton polytopes of polynomials from their toric Newton spectrum. We give some examples. 
1. Let

$$
\Delta:=\operatorname{conv}((0,0,0),(a, 0,0),(0, b, 0),(0,0, c))
$$

where $a, b$ and $c$ are positive integers. It is the Newton polytope of $f\left(u_{1}, u_{2}, u_{3}\right)=u_{1}^{a}+u_{2}^{b}+u_{3}^{c}$. Its toric Newton spectrum is the sequence of the following rational numbers:

- 0 ,

- $\frac{i}{a}$ for $1 \leq i \leq a-1, \frac{i}{b}$ for $1 \leq i \leq b-1, \frac{i}{c}$ for $1 \leq i \leq c-1$,

- $\frac{i}{a}+\frac{j}{b}$ for $1 \leq i \leq a-1$ and $1 \leq j \leq b-1, \frac{i}{a}+\frac{j}{c}$ for $1 \leq i \leq a-1$ and $1 \leq j \leq c-1$, $\frac{i}{b}+\frac{j}{c}$ for $1 \leq i \leq b-1$ and $1 \leq j \leq c-1$,

- $\frac{i}{a}+\frac{j}{b}+\frac{k}{c}$ for $1 \leq i \leq a-1,1 \leq j \leq b-1$ and $1 \leq k \leq c-1$.

Its $\delta$-vector is then given by Proposition 4.5. For instance, if $a=2, b=3$ and $c=3$ we get $\delta_{0}=1, \delta_{1}=10, \delta_{2}=7, \delta_{3}=0$. If $a=1, b=1$ and $c=1$ (this corresponds to the standard simplex in $\mathbb{R}^{n}$ ) we get $\delta_{0}=1, \delta_{1}=\delta_{2}=\delta_{3}=0$.

2. Let us consider the polytope in $\mathbb{R}^{3}$

$$
P:=\operatorname{conv}((0,0,0),(1,0,0),(0,1,0),(0,0,1),(1,1, h))
$$

where $h$ is an integer greater or equal to 2. This is a variation of Reeve's tetrahedron, see [2, Example 3.22]. It is the Newton polytope of $f\left(u_{1}, u_{2}, u_{3}\right)=u_{1}+u_{2}+u_{3}+u_{1} u_{2} u_{3}^{h}$ whose toric Newton spectrum is equal to $1+z+z^{2}+\sum_{i=1}^{h-1} z^{1+\frac{i}{h}}$. Thus, $\delta_{0}=1, \delta_{1}=1, \delta_{2}=h, \delta_{3}=0$ and

$$
L_{P}(z)=\frac{1}{6}\left[(h+2) z^{3}+9 z^{2}+(13-h) z+6\right] .
$$

Notice that the coefficient of $z$ is negative if $h \geq 14$.

\section{Reflexive polytopes and their Newton spectrum}

We give in this section various characterizations of reflexive polytopes involving their Newton spectrum. We show in particular that the spectrum of a polytope is equal to its $\delta$-vector if and only if $P$ is reflexive.

Proposition 5.1 Let $P$ be a polytope containing the origin in its interior and let $\delta_{P}(z)=\delta_{0}+$ $\delta_{1} z+\cdots+\delta_{n} z^{n}$ be its $\delta$-vector. Then the following are equivalent:

1. $\operatorname{Spec}_{P}(z)$ is a polynomial,

2. $P$ is reflexive,

3. $\operatorname{Spec}_{P}(z)=\delta_{P}(z):=\delta_{0}+\delta_{1} z+\cdots+\delta_{n} z^{n}$.

Proof. $1 \Leftrightarrow 2$. Assume that $P$ is reflexive: if $n$ belongs to the cone $\sigma_{F}$ supported by the facet $F$, the Newton function of $P$ is defined by $\nu: n \mapsto<u_{F}, n>$ where $u_{F} \in M$ by (3). By Proposition 2.4. $\operatorname{Spec}_{P}(z)$ is a polynomial. Conversely, assume that $\operatorname{Spec}_{P}(z)$ is a polynomial. Let $F$ be a facet of $P$. Again by Proposition 2.4, the Newton function $\nu$ of $P$ takes integral values on the fundamental 
domain $\square\left(\sigma_{F}\right)$. It follows that $\nu$ takes integral values on $\sigma_{F} \cap N$ and, by linearity, we get a $\mathbb{Z}$-linear map $\nu_{\sigma_{F}}: N_{\sigma_{F}} \rightarrow \mathbb{Z}$ where $N_{\sigma_{F}}$ is the sublattice of $N$ generated by the points of $\sigma_{F} \cap N$. Because we have the isomorphism $\operatorname{Hom}_{\mathbb{Z}}\left(N_{\sigma_{F}}, \mathbb{Z}\right) \cong M / \sigma_{F}^{\perp} \cap M$ induced by the dual pairing between $M$ and $N$, there exists $u_{F} \in M$ such that $\nu(n)=\left\langle u_{F}, n\right\rangle$ when $n \in \sigma_{F}$. In particular, the equation of $F$ is $\left\langle u_{F}, x\right\rangle=1$ for $u_{F} \in M$. Thus, $P$ is reflexive.

$1 \Leftrightarrow 3$. Assume that $\operatorname{Spec}_{P}(z)$ is a polynomial: by Proposition 4.5, $\delta_{i}=\operatorname{Card}\left\{\alpha \in \operatorname{Spec}_{P}, \alpha=i\right\}$ thus $\operatorname{Spec}_{P}(z)=\delta_{P}(z)$. Conversely, if $\operatorname{Spec}_{P}(z)=\delta_{P}(z)$ the Newton spectrum is obviously a polynomial.

Remark 5.2 Recall that a polynomial $a_{0}+a_{1} z+\cdots+a_{n} z^{n}$ is unimodal if there exists an index $j$ such that $a_{i} \leq a_{i+1}$ for all $i<j$ and $a_{i} \geq a_{i+1}$ for all $i \geq j$. It follows from Corollary 2.2, Proposition 5.1 and [11], [12] that the spectrum at infinity of a Laurent polynomial is not always unimodal (and this corrects a misguided assertion made in [6]). This can be directly checked: let

$$
\Delta:=\operatorname{conv}\left(e_{1}, \cdots, e_{9},-\sum_{i=1}^{9} q_{i} e_{i}\right)
$$

where $\left(e_{1}, \cdots, e_{9}\right)$ is the standard basis of $\mathbb{R}^{9}$ and $\left(q_{1}, \cdots, q_{9}\right):=(1, \cdots, 1,3)$ (this example is borrowed from [12], see also [3, Section 2]). Then $\mu_{\Delta}=12$ and $Q(\Delta)=(1, \cdots, 1,3)$ where 1 is counted 9-times: the simplex $\Delta$ is reduced and reflexive. We get from Theorem 3.3

$$
\operatorname{Spec}_{\Delta}(z)=1+z+z^{2}+2 z^{3}+z^{4}+z^{5}+2 z^{6}+z^{7}+z^{8}+z^{9}
$$

and this polynomial is not unimodal. It follows that the spectrum at infinity of the Laurent polynomial

$$
f\left(u_{1}, \cdots, u_{9}\right)=u_{1}+\cdots+u_{9}+\frac{1}{u_{1} \cdots u_{8} u_{9}^{3}}
$$

(see Section 2.2) is not unimodal.

Remark 5.3 Let $P$ be a reflexive polytope and let $\operatorname{Spec}_{P}(z)=\delta_{P}(z):=\delta_{0}+\delta_{1} z+\cdots+\delta_{n} z^{n}$ be its $\delta$-vector. The mean of the $\delta$-vector is $\frac{1}{\mu_{P}} \sum_{i=0}^{n} i \delta_{i}$ (and this is equal to $\frac{n}{2}$ because $\delta_{i}=\delta_{n-i}$ if $P$ is reflexive, as it could be deduced from Proposition 5.1) and its variance is $\frac{1}{\mu_{P}} \sum_{i=0}^{n} \delta_{i}\left(i-\frac{n}{2}\right)^{2}$. After Corollary [2.2, Proposition 5.1 and the version of Hertling's conjecture mentioned in the introduction (see [6] for details), the inequality $\frac{1}{\mu_{P}} \sum_{i=0}^{n} \delta_{i}\left(i-\frac{n}{2}\right)^{2} \geq \frac{n}{12}$ is expected.

\section{References}

[1] Batyrev, V.: Stringy Hodge numbers of varieties with Gorenstein canonical singularities, In: M-H Saito (ed.) et al., Integrable Systems and Algebraic Geometry, Proceedings of the 41st Taniguchi Symposium, Japan 1997, World Scientific, 1998, p. 1-32.

[2] Beck, M. , Robbins, S.: Computing the continuous discretely, Springer, New York, 2007.

[3] Braun, B., Davis, R., Solus, L.: Detecting the integer decomposition property and Ehrhart unimodality in reflexive simplices, Advances in Applied Math., 100, 2018, p. 122-142. 
[4] Conrads, H. : Weighted projective spaces and reflexive simplices, Manuscripta Math., 107 2002, p. 215-227.

[5] Douai, A.: A note on the Newton spectrum of a polynomial, arXiv:1810.03901.

[6] Douai, A.: Global spectra, polytopes and stacky invariants, Math. Z., 288 (3), 2018, p. 889913.

[7] Douai, A., Sabbah, C.: Gauss-Manin systems, Brieskorn lattices and Frobenius structures I, Ann. Inst. Fourier, 53 (4), 2003, p. 1055-1116.

[8] Douai, A., Sabbah, C.: Gauss-Manin systems, Brieskorn lattices and Frobenius structures II, In : Frobenius Manifolds, C. Hertling and M. Marcolli (Eds.), Aspects of Mathematics E 36 (2004).

[9] Hertling, C.: Frobenius manifolds and variance of the spectral numbers, In : New Developpments in Singularity Theory, D. Siersma, C.T.C Wall, V. Zakalyukin (Eds.), NATO Science Series (Series II: Mathematics, Physics and Chemistry), 21, Springer, Dordrecht.

[10] Kouchnirenko, A.G.: Polyèdres de Newton et nombres de Milnor, Invent. Math., 32, 1976, p. $1-31$.

[11] Mustaţă, M., Payne, S.: Ehrhart polynomials and stringy Betti numbers, Mathematische Annalen, 333(4), 787-795 (2005).

[12] Payne, S.: Ehrhart series and lattice triangulations, Discrete Comput. Geom., 40-3, 2008, p. 365-376.

[13] Sabbah, C.: Hypergeometric periods for a tame polynomial, Portugalia Mathematicae, 63, 2006, p. 173-226.

[14] Stapledon, A.: Weighted Ehrhart Theory and Orbifold Cohomology, Adv. Math., 219, 2008, p. $63-88$. 